\title{
MEASUREMENT OF SIBERIAN RADIOHELIOGRAPH CABLE DELAYS
}

\author{
S.V. Lesovoi \\ Institute of Solar-Terrestrial Physics SB RAS, \\ Irkutsk, Russia, svlesovoi@gmail.com
}

\author{
M.V. Globa \\ Institute of Solar-Terrestrial Physics SB RAS, \\ Irkutsk, Russia,globa@iszf.irk.ru
}

\begin{abstract}
To achieve the maximum dynamic range of solar radio images obtained using aperture synthesis in relatively wide frequency bands $0.1-0.5 \%$ of the operating frequency, it is necessary to compensate the signal propagation delays in the antenna receive path before calculating visibility functions (hereinafter visibilities). When visibilities are corrected without delay compensation, the signal-to-noise ratio decreases due to residual phase slopes in the receiving system bandwidth. In addition to enhancing dynamic range, preliminary compensation for delays simplifies real-time imaging no antenna gain calibration is required to get a first approximation image. The requirements for the accuracy of antenna placement are also reduced - in contrast to the measurement of the phase visibility error, the measurement of the delay is actually not so critical to the antenna position errors that are larger than the operating wavelength. The instantaneous frequency band of the Siberian Radioheliograph, which determines the minimum step for measuring the phase slope, and hence the accuracy of determining the delay, is $10 \mathrm{MHz}$. At the
\end{abstract}

speed of light in an optical fiber of $\sim 0.7 c$, a step of 10 $\mathrm{MHz}$ makes it possible to unambiguously measure the difference between electrical lengths of cables up to 20 $\mathrm{m}$ and to correct antenna positions by radio observations, even if the error in the position of the antennas exceeds the operating wavelength. Correction of the band phase slopes during the observation time adapts the radio telescope to the temperature drift of delays and decreases antenna gain phase spread. This, in turn, leads to more stable solutions to systems of equations containing antenna gains as unknowns.

Keywords: radio telescope, aperture synthesis, radio image.

\section{INTRODUCTION}

The quality of images obtained by radio telescopes using aperture synthesis significantly depends on calibrations of complex antenna gains. Without determining amplitude and especially phase errors in the antenna gains, we can get only a very rough image, and most often uncalibrated data cannot be considered as an image. A criterion of image quality is generally agreed to be its dynamic range - defined as the ratio of the brightest image detail to noise in the radiation-free image area. This is due to the fact that the residuals arising from image reconstruction (cleaning) are linearly related to errors in phases and amplitudes of visibilities [Perley, 1999]. Consider the residuals caused by phase errors in antenna gains:

$$
R_{\phi}=2 \Delta \phi \sin (2 \pi u l),
$$

where $\Delta \phi$ is the phase visibility error in radians, $u$ is the spatial frequency, $l$ is the direction cosine. If the errors are evenly distributed throughout all antennas, accordingly throughout all visibilities, the dynamic range of an image depends on phase errors as follows [Perley, 1999]:

$$
D=\frac{N}{\sqrt{2} \Delta \phi},
$$

where $N$ is the number of antennas in the antenna array. Note that this formula assumes that all $N(N-1) / 2$ visibil- ities of the $N$-antenna array are used for imaging. For SRH containing 48 antennas [Lesovoi et al., 2017], $N$ in (2) should be 32 rather than 48 due to the fact that only cross-visibilities are employed to construct an SRH image, not all possible visibilities. Their number is $2 \times 16 \times 32=1024$, which corresponds to $N=32$ in this formula. When observing solar flares, a dynamic range over $10^{4}$ is required to distinguish the solar disk. For example, at a maximum brightness temperature of $2.5 \cdot 10^{7} \mathrm{~K}$, a solar disk brightness temperature of $2 \cdot 10^{4}$ $\mathrm{K}$, and a 0.1 level of residuals relative to the solar disk, the phase error level should be no more than $0.05^{\circ}$, which corresponds the maximum difference between cable lengths $d l=c_{\mathrm{f}} \Delta \phi /(\pi \Delta v)=1 \mathrm{~cm}$, where $c_{\mathrm{f}}=0.7 \mathrm{c}$ is the speed of light in a fiber, $\Delta v=10 \mathrm{MHz}$ is the SRH operating frequency band. The required error level is comparable with the influence of non-factorable factors on visibility measurements and is not eliminated by generally accepted calibration methods. The cable length differences must therefore be compensated before calculating the visibility. An alternative would be to use a spectral correlator with an ultra-narrow frequency band of $\sim 0.002 \%$. In this case, it would be possible to compensate delays in each channel after measuring the visibility, and then to form the operating frequency band. There is no plan to develop a spectral correlator for $\mathrm{SRH}$ in the foreseeable future. In this paper, we propose 
an adaptive algorithm for correcting cable length differences before calculating visibilities in the correlator and report the results of applying such a correction to SRH signals.

\section{FORMULATION OF THE PROBLEM}

Let us consider the receive path of a solar radio interferometer, using the Siberian Radioheliograph (SRH) as an example [Altyntsev, 2020; Lesovoi et al., 2017; Lesovoi et al., 2012]. Signals from SRH antennas are transmitted to the working building via analog optical links. In the antenna module, a microwave signal modulates semiconductor laser power. Then, an optical signal is transmitted via a single-mode optical fiber to a photodetector, the signal at the output of which is proportional to the original microwave signal, delayed by the time of signal propagation in the optical fiber. When developing the SRH optical links, it was determined that lengths of all the links should be $375 \mathrm{~m}$. The equality between the lengths minimizes the effect of variance when transmitting signals from antennas to receivers, and the length of $375 \mathrm{~m}$ is sufficient to reach any of the SRH antennas. Measurements of cable lengths with the reflectometer have shown that a real difference between electrical cable lengths may be as great as $5 \mathrm{~m}$. With a difference between lengths $\Delta l=2 \div 5 \mathrm{~m}$ and the speed of light in the fiber $c_{\mathrm{f}}=0.7 c$, a decrease in the correlation coefficient will be $\operatorname{sinc}\left(\Delta v \Delta l / c_{\mathrm{f}}\right)=2 \div 10 \%$ [Walker, 1999], where $\Delta v$ is the SRH frequency band equal to 10 $\mathrm{MHz}$. To achieve the maximum dynamic range of SRH images, the difference in cable lengths must be corrected. The result of visibility measurement with such delays is affected by phase wrapping in the SRH full frequency band of $4 \mathrm{GHz}$. This leads to high phase noise when measuring frequency- and time-dependent visibilities. Moreover, different phase slopes in the antennas' operating frequency bands give rise to visibility errors that cannot be compensated by calibrations through antenna redundancy or by self-calibration [Cornwell, Fomalont, 1989]. The calibrations are reduced to any given method for solving a system of equations of the form [Thompson et al., 2017]

$$
\hat{V}_{k l}=g_{k} g_{l}^{*} G_{k l} V_{k l}+\epsilon_{k l}+n_{k l},
$$

where $\hat{V}_{k l}$ is the measured visibility, $g$ is antenna gains, $G_{k l}$ is the non-factorable antenna gain (unresolved into factors related to antennas), $V_{k l}$ is the true visibility, $\epsilon_{k l}$ is the additive correlator noise, $n_{k l}$ is the thermal noise. The usual way to solve system (3) is to reduce the influence of the terms $G_{k l}, \epsilon_{k l}, n_{k l}$ by hardware and to solve it as follows

$$
\hat{V}_{k l}=g_{k} g_{l}^{*} V_{k l} .
$$

Different phase slopes in channels of different antennas are manifested in the non-factorable part of the antenna gain $G_{k l}$, which does not allow us to reduce the number of unknowns in the system to the sum of the number of antennas and the number of visibilities, i.e. to make it smaller than the number of equations. Therefore, the calibration accuracy decreases. As indicated in
[Liu et al., 2010], with large phase errors in Equation (3) it is impossible to obtain an exact solution for antenna gains even in the absence of thermal noise. A change in the signal propagation delay in the optical fiber due to the temperature drift is $\sim 40-130 \mathrm{ps} /\left(\mathrm{km}^{\circ} \mathrm{C}\right)$. With daytime temperature drifts of several tens of degrees, typical for the sharply continental climate at the location of $\mathrm{SRH}$, a change in delay will be $\sim 1 \mathrm{~ns}$. For the highest SRH operating frequency of $8 \mathrm{GHz}$, this corresponds to eight periods. This is a very large value, even though it is only the difference in delay variations of different antennas that matters. It is therefore worthwhile to adapt the compensation for the delays in the path of each antenna to their variation in time.

A reflectometer can be used to measure cable lengths, but the accuracy achieved (with a not very expensive device) will not be higher than $10-20 \mathrm{~cm}$. Measuring the visibility phase slope in the SRH full frequency band enables us to measure relative cable lengths more accurately. In the SRH full operating frequency band $\Delta v_{\mathrm{RF}}=4 \mathrm{GHz}$, the difference between cable lengths $\Delta l=1 \mathrm{~cm}$ leads to a phase difference of $2 \pi \Delta v_{\mathrm{RF}} \Delta l / 0.7 c \approx 70^{\circ}$. The SRH sensitivity is sufficient to confidently approximate the phase slope of $70^{\circ} / 4 \mathrm{GHz}$ and hence to measure the electrical cable length up to 1 $\mathrm{cm}$, when estimating visibilities of the shortest antenna baselines. The compensation for the cable length difference, which tracks changes caused by the temperature drift, eliminates the problems arising when solving the systems of linear equations for phase calibration. One of the ways to linearize Equation (4) is to take the logarithm and to use the real part of the logarithm for amplitude calibration, and its imaginary part for phase calibration of antenna gains $g_{k}$. However, if the difference in cable lengths exceeds the wavelength, such linearization results in errors caused by ambiguity in the complex logarithm [Liu et al., 2010]. To successfully find a solution by the least square method, the following condition should be fulfilled

$$
\operatorname{Im}\left(\ln \left(\hat{V}_{k l}\right)\right)=\psi_{k l}+\phi_{k}-\phi_{l}< \pm \pi,
$$

where the imaginary part of the visibility logarithm is on the left, $\psi_{k l}$ is the true phase of visibility, $\phi_{k}, \phi_{l}$ are phases of the antenna gains $g_{k}, g_{l}$. Correcting the difference in cable lengths minimizes the right-hand side of (5), which makes phase calibration using redundancy more adequate. Perhaps, the most unpleasant consequence of unfulfilled (5) is the image shift caused by the time-dependent linear phase slope. Images in different polarizations are generally taken at different instants of time. Therefore, full intensity and circular polarization images are often distorted by different image shifts in left-hand and right-hand circular polarizations. To eliminate these distortions, it is necessary to center each image - to remove linear phase slopes from distributions of phase errors over antennas, which arise during calibration, or to use calibration algorithms that depend on the image model. When electrical cable lengths vary with time mainly due to temperature drifts in the refractive index of an optical fiber [Hartog et al., 1979], the most significant shifts occur at the moments when the 
difference in the electrical lengths is close to the operating wavelength. Let the visibility phase be determined only by the true visibility phase $\psi_{k l}$ and by delays in cables $\tau_{k}, \tau_{l}$ of respective antennas

$$
\theta_{k l}=\psi_{k l}+2 \pi v_{n}\left(\tau_{k}-\tau_{l}\right) .
$$

Consider the phase measurement with timedependent (for example, temperature-related) delays in the optical fiber. The measured visibility phase is

$$
\hat{\theta}_{k l}=\left(\psi_{k l}+2 \pi v_{n} \Delta \tau_{k l}(t)\right) \bmod 2 \pi,
$$

where $v_{n}$ is the operating frequency, $\Delta \tau_{k l}(t)$ is the timedependent delay. The rate of change of delay is determined by the rate of change of the environmental temperature at places where optical cables are laid. Experience shows that the corresponding change in the visibility phase by $2 \pi$ occurs for about an hour during periods of maximum environmental temperature drifts. To compensate for the phase errors caused by the temperature drift, it is necessary to correct phase slopes in antenna channels at least every half hour. The use of compensation for the phase errors caused by changes of delays in cables provides the real-time raw image without applying time consuming calculus for calibrating antenna gains. Any value is always measured with a finite precision. Without loss of generality, we can assume that the phase measurement accuracy depends on the nonmonotonicity of delay variation, i.e. in addition to the smooth delay variation owing to temperature there is delay noise $n_{\tau}$

$$
\hat{\theta}_{k l}=\left(\psi_{k l}+2 \pi v_{n} \Delta \tau_{k l}(t)+n_{\tau}(t)\right) \bmod 2 \pi .
$$

When $\Delta \tau_{k l}$ is close to an integer number of periods of the operating frequency $M / v_{n}$, the phase noise will be maximum due to $n_{\tau}(t)$ and division modulo. As mentioned above, visibilities in different circular polarizations are measured at different instants of time. Accordingly, the contribution of phase noise to the solution for phase distributions in different polarizations may be different. Equation (5) implies that in the case of a linear equidistant array for phases of excessive measured visibilities corresponding to the shortest antenna baselines the following relation holds:

$$
\hat{\theta}_{k, k+1}=\psi_{1}+\phi_{k}-\phi_{k+1},
$$

where $\psi_{1}$ is the phase obtained at the shortest baseline (the phase of the first harmonic of the spatial spectrum measured by SRH). The given vector is $\overrightarrow{\hat{\theta}}=\left[\hat{\theta}_{k, k+1}\right]$, and the required vector is $\vec{\phi}=\left[\psi_{1}, \phi_{1}, \ldots, \phi_{N}\right]$. The vector length $\overrightarrow{\hat{\theta}}$ is by 2 less than $\vec{\phi}$, therefore corresponding system of equations (9) is underdetermined and can be solved by the least square method. From (9) it follows that the solution for antenna gain phases can be expected to take the form

$$
\phi_{n}=\Delta \psi_{1}(n-1)+\Delta \phi_{1}-\sum_{k=1}^{n} \hat{\theta}_{k},_{k+1},
$$

where $n$ is the antenna number within $1, \ldots, N ; \Delta \psi_{1}$ is the error in finding the phase of the first harmonic, $\Delta \phi_{1}$ is the error in finding the phase of the first antenna. The factor multiplying $n$ determines the linear slope of phase distribution. The phase slope error, and hence shifts between images in different polarizations, is determined by the error in measuring the visibility phase. Consider solution (9) for a linear equidistant array containing 32 antennas $\overrightarrow{\hat{\theta}}=\mathbf{M} \vec{\phi}$, where $\mathbf{M}$ is a matrix with dimensions $[33,31]$ describing the transformation $\vec{\phi}$ in $\overrightarrow{\hat{\theta}}$. In general, the solution is $\vec{\phi}=\mathbf{P} \overrightarrow{\hat{\theta}}$, where $\mathbf{P}=\left(\mathbf{M}^{T} \mathbf{M}\right)^{-1} \mathbf{M}^{T}$ is the pseudoinverse matrix for $\mathbf{M}$. We have defined above the vector $\vec{\phi}$ such that the phase of true visibility is the first element of this vector. Then the estimated phase of the true visibility is determined by the dot product of the first row $\mathbf{P}$ and the vector containing phases of measured visibilities $\psi_{1}=\mathbf{P}_{0} \overrightarrow{\hat{\theta}}$. To figure out what defines $\Delta \psi_{1}$, it is necessary to show how $\psi_{1}$ is estimated. Since the solution to (9) by the least square method is reduced to multiplying the pseudoinverse matrix and the vector of measured visibilities, and for a given antenna array the pseudoinverse matrix is a constant, we can explore what exactly $\mathbf{P}_{0}$ is. If we approximate the dependence of the $\mathbf{P}_{0}$ elements on the element number by a second order polynomial, we get $\mathbf{P}_{0}$, $k=0.00567974+0.00549652 k-0.00018322 k^{2}$, where $k$ is the number of the vector element $\mathbf{P}_{0}$ or $\overrightarrow{\hat{\theta}}$. This means that the estimated $\psi_{1}$ is a weighted average of $\overrightarrow{\hat{\theta}}$. The deviation of the estimated $\psi_{1}$ from the true visibility phase depends on the phase noise level, and the effect of antenna noise on the deviation is the stronger, the closer the antenna is to the center of the array.

It follows from the above that the algorithm of adaptive correction of length differences of cables transmitting signals from SRH antennas to the working building should provide more stable solutions obtained during phase correction of measured visibilities.

\section{REALIZATION}

The phase slope corresponding to the signal propagation delay in the optical fiber is measured by estimating the visibility phase slope of the SRH shortest antenna baseline. In this case, the decisive contribution to the visibility phase is assumed to be made by the response to the solar disk, and the linear phase slope is caused by the delay in the optical fiber, rather than by solar activity. Indeed, as mentioned above, the $1 \mathrm{~cm}$ difference between cable lengths leads to a $\sim 70^{\circ}$ change of the visibility phase in the SRH full frequency band. To obtain such a phase change due to the asymmetric location of the solar activity source, the microwave radiation source, located on the solar limb, should have a flux density by an order of magnitude higher than the solar disk flux density, or such a source should have a flux density comparable to the disk flux density at a minimum spatial frequency and increasing by about two orders of magnitude at a maximum spatial frequency. 
For the quiet Sun in the absence of flares, this is highly unlikely.

The result of the linear regression of the visibility phase-frequency dependence is used as the linear slope. One should remember that the Sun is an extended source; therefore, its spatial spectrum distorts this dependence. The spatial spectrum of the quiet Sun is close to the Bessel function of the first kind divided by its argument. Therefore, the spectrum contains minima, where the visibility modulus drops to almost zero. In passing through the minimum when falling on another spectrum lobe, the visibility phase undergoes a jump by $\pi$. These features can distort the phase-frequency dependence and lead to an incorrect estimate of the delay. The delay is estimated most accurately when visibilities of a given baseline at all frequencies do not go beyond one of the spatial spectrum lobes.

As mentioned above, the SRH cable length difference $\Delta l$, according to the estimates provided by the reflectometer, may be as large as $2-5 \mathrm{~m}$. The primary measurement of phase slopes should therefore be made with a reduced frequency step $\delta v<c_{f} / \Delta l$ so that the phase values do not go beyond $2 \pi$ in one step. After introducing the primary correcting delays into the receive path, the frequency step may be increased. Experience shows that these primary measurements are unambiguous at a $50 \mathrm{MHz}$ step, while a $100-200 \mathrm{MHz}$ step is usually used in observations. The procedure for introducing changes into delays should be cumulative: the current difference between lengths is always measured taking into account the introduced delays. The result of the real-time correction of phase slopes in the SRH receive path is presented in the figure. Note that such a correction provides a more stable calibration during further processing of SRH data. By reducing the phase noise, it is easier to solve the problem of overlapping images captured in different polarizations.

To correct the phase slope, we need to use a combination of delay lines, fractional signal shifts (for a time less than the sampling period), and phase control of digital local oscillators. Suppose that a signal is received in the upper sideband at a frequency $v_{\mathrm{USB}}$, the delay $\Delta \tau$ is measured in picoseconds, the signal sampling rate is $100 \mathrm{MHz}$, and the step of changing the signal delay at the output of an interpolating filter is $0.1 \mathrm{~ns}$.

A step of adjusting the digital local oscillator phase
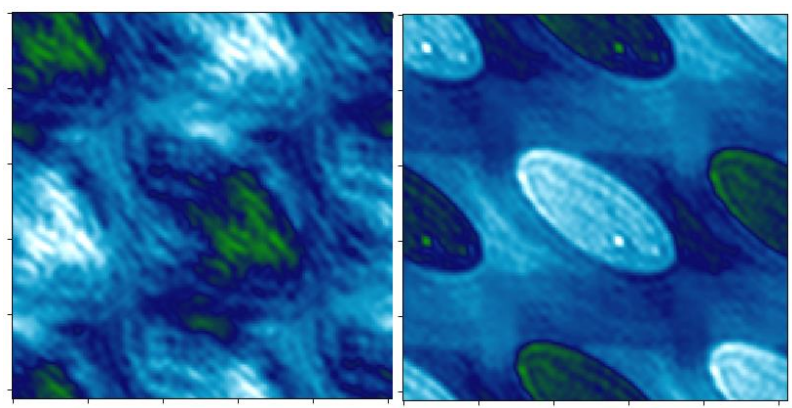

Result of consideration of the difference between SRH cable lengths. The images were taken in real time without phase calibration in the coordinate system of direction cosines. On the left is an image without correction of the length difference; on the right, with correction of the lengths (phase slopes)
$\Delta \varphi=0.3^{\circ}$. The processor must convert a given delay $\Delta \tau$ to $\Delta \tau_{n}=N_{1} 10^{4}+N_{2} 10^{2}$ and $\Delta \phi_{n}=1.2 \cdot 10^{-9}(\Delta \tau-\Delta \tau \mathrm{n}) v_{\mathrm{USB}}$, where $N_{1}, N_{2}$ are the number of sampling frequency periods and the step of the interpolating filter respectively.

The phase errors introduced by such a correction are the smaller the further away both antennas are from the phase center of the array. The introduced phase noise, taking into account the fact that for each baseline the adjustment is made in both antennas many times during the accumulation time (distant antennas), is determined in a similar way [Thompson, 2007, http: //www.ovsa.njit.edu/wiki/index.php/Calibration_Overvi ew, ftp://ftp.rao.istp.ac.ru/SRH/FASR/Thompson_Delay _Fringe_Phsw.pdf], $\Delta \phi / 6 \approx 0.12^{\circ}$. For pairs in which both antennas are located close to the phase center of the array, a delay varies slowly with respect to the accumulation time. The phase noise (more precisely, phase jumps) may therefore be equal to $2 \Delta \phi$. To average the noise for all visibilities, the phase center of the antenna array should be located at the edge or even outside the array rather than at its geometric center. Realization of the phase center offset implies that certain constant values must be added to all time-varying values of geometric antenna delays.

\section{CONCLUSION}

We have proposed an algorithm for measuring and compensating delays in antenna channels of the Siberian Radioheliograph, adaptive to variations in electrical cable lengths, caused by a temperature drift. The delays are measured using the frequency dependence of the phase of measured visibility functions of redundant antenna pairs. This algorithm can compensate in real time phase errors in a path, arising from changes in electrical lengths of optical cables, and can provide a primary image without applying antenna gain calibration algorithms (see the figure). On the other hand, the phase slope compensation improves the signal-to-noise ratio in visibility measurements and results in more stable phase calibrations in further image processing.

The work was performed under Government Assignment for 2021 No. 075-00374-21-00 dated December 24, 2020 "Methods and Instruments of an Astrophysical Experiment" (unique number 0278-2021-0010, registration number CITiS 121040600115-2).

\section{REFERENCES}

Altyntsev A.T., Lesovoi S.V., Globa M.V., Gubin A.V., Kochanov A.A., Grechnev V.V., et al. Multiwave Siberian Radioheliograph. Solar-Terr. Phys. 2020, vol. 6, iss. 2, pp. 3040. DOI: $10.12737 /$ stp-62202003.

Cornwell T., Fomalont E.B. Self-calibration. Synthesis imaging in radio astronomy. A collection of Lectures from the Third NRAO Synthesis Imaging Summer School. Astronomical Society of the Pacific Publ., 1989, vol. 6, p. 185.

Hartog A.H., Conduit A.J., Payne D.N. Variation of pulse delay with stress and temperature in jacketed and unjacketed optical fibres. Optical and Quantum Electronics. 1979, vol. 11, pp. 265-273 DOI: 10.1007/BF00620112.

Lesovoi S.V., Altyntsev A.T., Ivanov E.F. Gubin A.V. The Multifrequency Siberian Radioheliograph. Solar Phys. 2012, vol. 280, iss. 2, pp. 651-661. DOI: 10.1007/s11207-012-0008-7. 
Lesovoi S.V., Altyntsev A.T., Kochanov A.A., Grechnev A.A., Gubin A.V., Zhdanov D.A., et al. Siberian Radioheliograph: first results. Solar-Terr. Phys. 2017, vol. 3, iss. $1, \quad$ pp. $3-18$. DOI: 10.12737/article_58f 96 ec60fec 52.86165286 .

Liu A., Tegmark M., Morrison S. Lutomirski A., Zaldarriaga M. Precision Calibration of Radio Interferometers Using Redundant Baselines. 2010. arXiv: 1001.5268 DOI: 10.1111/ j.1365-2966.2010.17174.x.

Perley R.A. High dynamic range imaging. Synthesis Imaging in Radio Astronomy II. A Collection of Lectures from the Sixth NRAO/NMIMT Synthesis Imaging Summer School. ASP Conference Ser. 1999. Vol. 180. P. 275. DOI: 1999ASPC..180..275P.

Thompson A.R. Delay tracking, fringe rotation, and phase switching in FASR. FASR memo. 2007.

Thompson A.R., Moran J.M., Swenson Jr. G.W. Interferometry and Synthesis in Radio Astronomy. Third Edition. Springer, 2017. DOI: 10.1007/978-3-319-44431-4.

Walker R.C. Very Long Baseline Interferometry. Synthesis Imaging in Radio Astronomy II. A Collection of Lectures from the Sixth NRAO/NMIMT Synthesis Imaging Summer School. ASP Conference Ser. 1999, vol. 180, p. 433.

URL: http://www.ovsa.njit.edu/wiki/index.php/Calibration Overview (accessed March 20, 2021).

URL: ftp://ftp.rao.istp.ac.ru/SRH/FASR/Thompson_Delay _Fringe_Phsw.pdf (accessed March 20, 2021).

How to cite this article

Lesovoi S.V., Globa M.V. Measurement of Siberian Radioheliograph cable delays. Solar-Terrestrial Physics. 2021. Vol. 7. Iss. 4. P. 93-97. DOI: $10.12737 /$ stp-74202110 